\title{
Mediators and moderators of the effects of a year-long exercise intervention on endogenous sex hormones in postmenopausal women
}

\author{
Christine M. Friedenreich · Heather K. Neilson • Christy G. Woolcott • \\ Qinggang Wang · Yutaka Yasui • Rollin F. Brant · Frank Z. Stanczyk • \\ Kristin L. Campbell $\cdot$ Kerry S. Courneya
}

Received: 16 March 2011/Accepted: 21 June 2011/Published online: 6 July 2011

(C) The Author(s) 2011. This article is published with open access at Springerlink.com

\begin{abstract}
Objective To identify factors that mediate or moderate the effects of exercise on postmenopausal sex hormone concentrations.

Methods Postmenopausal women were randomized to 12 months of aerobic exercise for $200 \mathrm{~min} /$ week $(n=160)$
\end{abstract}

Trial registration: Clinicaltrials.gov identifier: NCT00522262.

C. M. Friedenreich $(\bowtie) \cdot$ H. K. Neilson · Q. Wang

Department of Population Health Research, Alberta Health

Services-Cancer Care, Calgary, AB T2N 4N2, Canada

e-mail: christine.friedenreich@albertahealthservices.ca

C. G. Woolcott

Departments of Obstetrics and Gynaecology and Pediatrics,

Dalhousie University, Halifax, NS, Canada

Y. Yasui

Department of Public Health Sciences, University of Alberta,

Edmonton, AB, Canada

R. F. Brant

Department of Statistics, University of British Columbia,

Vancouver, BC, Canada

\section{F. Z. Stanczyk}

Department of Obstetrics and Gynecology, University of

Southern California, Keck School of Medicine, Los Angeles,

CA, USA

\section{K. L. Campbell}

Department of Physical Therapy, University of British

Columbia, Vancouver, BC, Canada

\section{K. S. Courneya}

Faculty of Physical Education and Recreation, University of

Alberta, Edmonton, AB, Canada or to a control group $(n=160)$. Intention-to-treat analyses were performed using general linear models with sex hormone concentrations at 6 and 12 months as the outcome. Mediation by adiposity and insulin was investigated by examining changes in effect estimates after adjustment for changes in these factors over 12 months. Moderation was studied as the interaction between group assignment and eight baseline characteristics.

Results Intervention effects on sex hormone-binding globulin (SHBG) and estradiol changes were attenuated with adjustment for change in overall body fat, while there was less attenuation adjusting for intra-abdominal fat change. Intervention effects on SHBG levels were unaffected by adjustment for insulin change. Significant interactions were identified between treatment and physical fitness (for SHBG and testosterone) and age (for testosterone), implying subgroup differences in intervention effect. Conclusions Our data suggest that overall fat loss partially mediated exercise-induced changes in estradiol and SHBG concentrations. No previous RCT in postmenopausal women has studied moderators of exercise-induced sex hormone changes; therefore, future studies are needed to corroborate our results.

Keywords Exercise - Gonadal steroid hormones . Sex hormone-binding globulin $\cdot$ Randomized controlled trial $\cdot$ Breast neoplasms

\section{Introduction}

A wealth of epidemiologic evidence supports a preventive role for physical activity in postmenopausal breast cancer etiology, but the biologic changes mediating this 
association remain poorly understood [1,2]. Two plausible mechanisms are a decrease in sex hormone concentrations and an increase in sex hormone-binding globulin (SHBG) concentrations, which are convincingly associated with postmenopausal breast cancer risk [3]. As adipose tissue is the main source of sex hormones in postmenopausal women, exercise-induced fat loss could drive changes in their levels [4]. Exercise-induced reductions in insulin could increase SHBG synthesis in the liver [5].

It is also unclear whether any factors moderate the association between physical activity and postmenopausal breast cancer risk. Although the epidemiologic evidence suggests that the relationship between physical activity and reduced postmenopausal breast cancer risk is probable $[1,2]$, there is variability across studies in the magnitude of the association, possibly attributable to subgroup differences in the study populations defined by factors such as age and BMI [1]. These factors, and others, may also modify the effects of physical activity on sex hormone or SHBG levels, perhaps because they relate to baseline levels of sex hormones and SHBG (high baseline levels may result in a ceiling effect whereby exercise will not result in further increase; low baseline levels may result in a floor effect whereby exercise will not result in further decrease) or because they relate to exercise adherence.

The Alberta Physical Activity and Breast Cancer Prevention (ALPHA) Trial was a randomized controlled trial (RCT) designed to determine the effects of a 12-month aerobic exercise intervention on proposed biomarkers of postmenopausal breast cancer risk. We previously reported that, relative to controls, postmenopausal women assigned to the intervention experienced significantly greater decreases in circulating total and free estradiol [6], circulating insulin [7], and total and abdominal fat [8] and significantly greater increases in SHBG levels [6]. Because clarifying the biologic mechanisms through which exercise induces sex hormone and SHBG changes and distinguishing subgroups of women in whom exercise may have a stronger effect on sex hormone and SHBG changes could help inform public health recommendations for lowering postmenopausal breast cancer risk, we present here a secondary analysis of ALPHA Trial data using analytic techniques described by Kraemer et al. [9] and Mackinnon and Fairchild [10] to (1) explore adiposity and insulin changes as potential mediators of the exercise-induced estradiol and SHBG changes we observed and (2) examine baseline characteristics as potential moderators of the intervention effect on changes in estradiol, estrone, testosterone, and SHBG, which are all convincingly associated with postmenopausal breast cancer risk [3].

\section{Materials and methods}

Study population

The ALPHA Trial study population and recruitment details have been described previously [6, 8]. Inactive women ( $<90 \mathrm{~min} /$ week recreational activity over the past year or, if between 90 and $120 \mathrm{~min} /$ week of physical activity had a $\mathrm{VO}_{2 \max }<34.5 \mathrm{~mL} / \mathrm{kg} / \mathrm{min}$ ), aged $50-74$ years, postmenopausal for at least 24 months, body mass index (BMI) $22-40 \mathrm{~kg} / \mathrm{m}^{2}$, with no major co-morbidities or previous cancer diagnosis besides non-melanotic skin cancer, were recruited from the general population in Calgary and Edmonton, Alberta, Canada. Eligibility criteria also included the following: English-speaking, acceptable heart and lung function as assessed by a baseline fitness test, able to undertake unrestricted physical activity as assessed by their physician [11], normal levels of fasting lipids, glucose, thyroid-stimulating hormone and alanine aminotransferase, breast tissue density above a zero density level, non-diabetic, non-smoker, alcohol intake $<14$ drinks/week, no medications or exogenous hormones that might influence estrogen metabolism and not currently or planning to undertake a weight loss program or planning any extended absences in the 18 months subsequent to enrollment.

\section{Intervention}

The study participants were randomly allocated either to a year-long, moderate-to-vigorous-intensity, aerobic exercise intervention $(n=160)$ or to a control group $(n=160)$ assigned to no exercise. Details of randomization have been described previously [6]. The exercise intervention was individually prescribed program of at least 45 min of any type of aerobic exercise done 5 days per week for 12 months at 70-80\% heart rate reserve. At least three sessions per week were supervised by exercise trainers at our fitness centers, and the rest were home-based. The frequency, duration, and intensity were gradually increased during the first 3 months of the intervention from 3 weekly sessions of 15 - to 20-min duration at an intensity of $50-60 \%$ heart rate reserve to the final prescription achieved in week 12 . Women in the control group were asked to maintain their regular inactive lifestyle. No changes to usual dietary intake were to be made in either group. Ethics approval was obtained from the Alberta Cancer Board and Universities of Calgary and Alberta, and all participants provided written informed consent.

\section{Measurements}

Baseline data on demographics, medical and medication history, self-rated health, and past year physical activity 
[12] were obtained by a self-administered questionnaire. Anthropometric measurements were taken in duplicate; if the two measurements were discrepant (i.e., not identical), a third measurement was taken and the average of the two closest was used in the analyses. Weight and height measurements were taken using a balance beam scale and a stadiometer. Body mass index was calculated as weight/ height $^{2}\left(\mathrm{~kg} / \mathrm{m}^{2}\right)$. At baseline and 12 months, total body fat and body fat percentage were measured using whole-body dual X-ray absorptiometry scans. In Calgary, scans were done on a Hologic QDR $4500 \mathrm{~W}^{\circledR}$ scanner in whole-body mode and analyzed with software version 11.2.1 (Hologic ${ }^{\circledR}$, Inc., Bedford, MA, USA). In Edmonton, scans were done on a Lunar Prodigy ${ }^{\circledR}$ scanner in either standard mode or thick mode and analyzed with enCORE Software 6.70.01 before November 2004 and 8.60 after November 2004 (Lunar ${ }^{\circledR}$ General Electric Medical Systems, Madison, WI, USA). Percent body fat was calculated as $100 \% \times\{$ fat mass/(fat mass + lean mass) $\}$. Intra-abdominal fat was measured with a single computed tomography slice at the umbilicus. In Calgary, scans were done using a Marconi PQ5000 VisionMaster/Picker, and in Edmonton, using a Phillips/Marconi MX8000 multislice scanner. The study radiologist used an image analysis software program (Phillips Medical Systems PQ5000) to identify and demarcate the thresholds between the subcutaneous and intra-abdominal area. Physical fitness was assessed at baseline and 12 months using a modified Balke treadmill protocol to estimate maximum oxygen consumption $\left(\mathrm{VO}_{2 \max }\right)$ from submaximal exercise intensities. Oxygen consumption at the age-predicted maximum heart rate was estimated by extrapolating from the two last completed stages using the American College of Sports Medicine metabolic equations for estimating oxygen consumption at the workload of each stage [13].

Blood was collected after a minimum 10-h fast at baseline $(60 \mathrm{~mL}), 6$ and 12 months $(40 \mathrm{~mL})$, and medications taken in the past $24 \mathrm{~h}$ were recorded. Participants were asked not to exercise for $24 \mathrm{~h}$ pre-blood draw. All blood samples were collected, processed, and stored within $12 \mathrm{~h}$ of collection and then shipped and stored in $-86^{\circ} \mathrm{C}$ freezers until time of assay. Analyses were conducted by the Reproductive Endocrine Research Laboratory at the University of Southern California, a laboratory with wellestablished protocols and quality control procedures. Serum estradiol and testosterone levels were quantified by radioimmunoassay after organic solvent extraction and Celite column partition chromatography [14-16]. Chromatographic separation of the steroids was achieved by using different concentrations of toluene in iso-octane and ethyl acetate in iso-octane. Sex hormone-binding globulin (SHBG) and insulin were quantified via chemiluminescent immunometric assay using the Immulite Analyzer
(Siemens Healthcare Diagnostics, Deerfield, IL). The SHBG concentration and an assumed albumin concentration of $43 \mathrm{~g} / \mathrm{L}$ were then utilized in a validated algorithm with total estradiol to calculate free estradiol [17].

Hypothesized mediators and moderators

Our hypothesized mediators were body fat change (for sex hormones and SHBG) and circulating insulin change (for SHBG). These hypotheses were supported by our earlier review of the scientific literature on proposed biologic mechanisms relating physical activity to postmenopausal breast cancer risk [4]. Moreover, the proposed mediators, by definition [9], were associated with our exercise intervention; relative to controls, the exercise group in the ALPHA Trial experienced significantly greater decreases in total body fat, intra-abdominal fat area [8], and circulating insulin levels [7] over 12 months.

Potential moderators were baseline characteristics for ALPHA Trial participants that were selected for their hypothetical relations either to exercise adherence during the trial (e.g., baseline fitness level, age, years postmenopause, self-rated health, previous recreational activity, baseline BMI) or to changes in sex hormone levels (e.g., circulating levels of sex hormones at baseline, previous HRT use, baseline BMI). We hypothesized that women who exercised more might experience greater changes in sex hormone levels and that women with higher sex hormone levels or lower SHBG levels at baseline might experience greater changes as a result of the exercise intervention.

\section{Statistical analyses}

The analyses included participants with complete data and were based on assigned groups at randomization regardless of adherence. Hormone levels were log-transformed to achieve an approximate symmetric distribution. Intervention effects were evaluated with general linear models considering the repeated measures of sex hormone concentrations at 6 and 12 months. The model took the following form: $Y_{i j}=\beta_{0}+\beta_{1} X_{i}+\gamma Z_{i j}+\varepsilon_{i j}$, where, for the $i$ th subject, $Y_{i j}$ is a log-transformed outcome measure at $j$ th time ( $j=1$ for 6 months, $j=2$ for 12 months), $X_{i}$ is an indicator variable for the exercise intervention $\operatorname{arm}(0$ for control, 1 for exercise intervention), $\boldsymbol{Z}_{\boldsymbol{i} \boldsymbol{j}}$ is a vector of adjustment variables, and $\left(\boldsymbol{\varepsilon}_{i 1}, \boldsymbol{\varepsilon}_{i 2}\right)$ is a correlated error vector following a bivariate Gaussian distribution with mean 0 . Of the parameters $\beta_{0}, \beta_{1}$, and $\gamma$ (associated with the intercept, exercise intervention, and adjustment variables, respectively), $\beta_{1}$ is the target parameter, representing the adjusted difference in the mean log outcome between the exercise intervention group and the control group over 
the two follow-up time points, adjusting for $\boldsymbol{Z}$. We refer to the exponential of $\beta_{1}$ as the treatment effect ratio (TER), as it is a ratio of the adjusted geometric means of the outcome for the exercise intervention group over the control group. In this paper, we also refer to the TER as the 'intervention effect'.

Our assessment of mediation was based on findings from the general linear model with both the intervention assignment and changes in the hypothesized mediators predicting changes in sex hormone or SHBG levels. Mediation by adiposity and insulin levels was investigated in two ways. Primarily, mediation was investigated by examining the change in the TER pre- versus postadjustment for changes in the hypothesized mediators at 6 and 12 months from baseline. If the adjustment attenuated TER, then we considered this finding to be suggestive of mediation [10]. Secondarily, the main effect of each potential mediator on sex hormone or SHBG changes was also evaluated in these models, adjusting for the intervention assignment. Kraemer et al. [9] explain that a mediator must measure a change occurring during treatment (e.g., changes in body fat and insulin levels occurred during the 12-month intervention period [7, 8]), must correlate with treatment assignment (e.g., we observed significantly greater changes in body fat and insulin levels in the exercise group than in controls [7, 8]), and must have either a main or interactive effect on the outcome. This secondary analysis is informative because it is these conditions under which our main analysis would suggest mediation.

Moderation was investigated by determining the statistical significance of the interaction term between the intervention assignment and each hypothesized moderator (described above), adjusting for the baseline outcome (e.g., baseline estradiol level), the intervention group assignment, time, and the hypothesized moderator (e.g., baseline age) [9]. All baseline characteristics were treated as continuous variables in these analyses with the exception of HRT use, which was dichotomized. To help explain our findings from these analyses, intervention effects were also estimated within subgroups of these characteristics. We reported intervention effects within all subgroups regardless of the statistical significance of the interaction term in general linear models. Subgroups were created by dichotomizing each variable at the median with the exception of self-rated health, which was measured by questionnaire using the SF-36 scale [18] (range, $0-100$ ): 'low' was defined as a score $<82$ and $\geq 82$ was considered 'high'. All statistical tests were two-sided with a significance level set at 0.05 . Statistical analyses were performed using SAS software (version 9.1; SAS Institute, Inc., Cary NC).

\section{Results}

The flow of participants through the trial has been reported elsewhere [6]. Briefly, we assessed 3,454 women for eligibility and 320 women were randomized with 165 women from Edmonton and 155 from Calgary. We had nine women withdraw from the study and provide no follow-up data. For these analyses, we had 153 exercisers and 154 controls with complete data. Descriptive statistics for baseline and 12-month levels of sex hormones, SHBG and insulin levels, and all anthropometric measures have been reported previously [6-8], and only a summary is provided here (Table 1).

Women randomized to the exercise and control arms were similar on all demographic, anthropometric, and lifestyle characteristics. Our study population had a mean age of 61 years, were overweight (mean BMI $=29$ ), relatively healthy and fit $\left(\mathrm{VO}_{2 \max }\right.$ was $\left.27 \mathrm{~mL} / \mathrm{kg} / \mathrm{min}\right)$, inactive (11 MET-hours/week of recreational activity in the year prior to the study), mainly Caucasian (91\% white), married (74\%), and fairly well educated (67\% greater than high school education).

\section{Mediation}

In the present analysis, adjustment for change in percent and total body fat attenuated intervention effects on total estradiol concentrations, but change in intra-abdominal fat area did not (Table 2). For free estradiol, the attenuation was slightly stronger with adjustment for overall body fat change than for intra-abdominal fat change. Intervention effects on SHBG change were attenuated to almost null with adjustment for total and percent body fat and attenuated to a slightly lesser extent with adjustment for intraabdominal fat change (Table 2). Adjusting for insulin change did not influence the intervention effect. In these same models, however, insulin change was associated with SHBG change when adjusting for intervention effects ( $p$ for insulin $=0.001)$. In exploratory analyses, SHBG change and insulin change were significantly inversely correlated in controls $(p=0.003)$, but not in exercisers $(p=0.304)$.

\section{Moderation}

The statistical significance of the interaction term between intervention group and each potential moderator is shown in Table 3.

No statistically significant moderation by baseline characteristics was found for estradiol and estrone changes. For SHBG changes, the intervention effect was statistically significantly higher among women with lower baseline 
Table 1 Baseline characteristics of randomized participants in the ALPHA

Trial, Alberta, Canada, $2003-2007, n=320$

\begin{tabular}{lcc}
\hline Baseline characteristics & $\begin{array}{c}\text { Exercisers }(n=160) \\
\text { Mean } \pm \text { SD }\end{array}$ & $\begin{array}{c}\text { Controls }(n=160) \\
\text { Mean } \pm \text { SD }\end{array}$ \\
\hline Age (yrs) & $61.2 \pm 5.4$ & $60.6 \pm 5.7$ \\
Body composition measurements & $29.1 \pm 4.5$ & \\
Body mass index $\left(\mathrm{kg} / \mathrm{m}^{2}\right)$ & $101.4 \pm 55.4$ & $29.2 \pm 4.3$ \\
Intra-abdominal fat area $\left(\mathrm{cm}^{2}\right)$ & $30.9 \pm 8.2$ & $103.2 \pm 56.0$ \\
Total body fat $(\mathrm{kg})$ & $42.2 \pm 4.9$ & $31.3 \pm 8.6$ \\
Percent body fat & $n(\%)$ & $42.4 \pm 5.7$ \\
\hline & $82(55)$ & $n(\%)$ \\
\hline Full-time employment & $112(70)$ & $102(64)$ \\
Education $(>$ high school) & Median (IQR) & Median (IQR) \\
\hline & & $10.0(7.0-14.0)$ \\
\hline Sex hormone concentration & $9.0(7.0-12.0)$ & $32.0(22.5-44.5)$ \\
Estradiol $(\mathrm{pg} / \mathrm{mL})$ & $32.0(24.0-44.0)$ & $39.5(28.0-51.5)$ \\
Estrone (pg/mL) & $41.5(30.0-57.0)$ & $547(417-736)$ \\
Sex hormone-binding globulin (nmol/L) & $576(434-779)$ & $23.3(17.5-32.4)$ \\
Androstenedione $(\mathrm{pg} / \mathrm{mL})$ & $24.3(16.8-32.8)$ & $0.24(0.18-0.36)$ \\
Testosterone $(\mathrm{ng} / \mathrm{dL})$ & $0.23(0.17-0.31)$ & $0.36(0.25-0.49)$ \\
Free estradiol $(\mathrm{pg} / \mathrm{mL})$ & $0.36(0.24-0.48)$ & \\
Free testosterone $(\mathrm{ng} / \mathrm{dL})$ & $6.2(3.8-9.5)$ & \\
Metabolic hormone concentration & & \\
Insulin ( $\mu \mathrm{IU} / \mathrm{mL})$ & & \\
\hline
\end{tabular}

Table 2 Exercise intervention effects on estradiol and sex hormone-binding globulin before and after adjustment for hypothesized mediators

\begin{tabular}{|c|c|c|c|c|}
\hline \multirow[t]{2}{*}{ Outcome } & \multirow{2}{*}{$\begin{array}{l}\text { Before adjustment } \\
\text { Treatment effect ratio } \\
\text { of Exercise/Control }(95 \% \mathrm{CI})^{\text {a }}\end{array}$} & \multirow{2}{*}{$\begin{array}{l}\text { Hypothesized } \\
\text { mediator }\end{array}$} & \multicolumn{2}{|l|}{ After adjustment } \\
\hline & & & $\begin{array}{l}\text { Treatment Effect Ratio } \\
\text { of Exercise/Control }(95 \% \mathrm{CI})^{\text {a }}\end{array}$ & $p$ for mediator ${ }^{\mathrm{b}}$ \\
\hline \multicolumn{5}{|l|}{ Estradiol } \\
\hline & \multirow{3}{*}{$0.93(0.88-0.98)$} & Percent body fat & $0.95(0.90-1.00)$ & 0.005 \\
\hline & & Total body fat & $0.95(0.90-1.00)$ & 0.005 \\
\hline & & Intra-abdominal fat area & $0.93(0.89-0.99)$ & 0.176 \\
\hline \multicolumn{5}{|c|}{ Sex hormone-binding globulin } \\
\hline & \multirow[t]{4}{*}{$1.04(1.02-1.07)$} & Percent body fat & $1.01(0.98-1.03)$ & $<.001$ \\
\hline & & Total body fat & $1.01(0.98-1.03)$ & $<.001$ \\
\hline & & Intra-abdominal fat area & $1.02(0.99-1.05)$ & $<.001$ \\
\hline & & Insulin & $1.04(1.01-1.06)$ & 0.001 \\
\hline \multicolumn{5}{|c|}{ Free estradiol } \\
\hline & \multirow[t]{3}{*}{$0.91(0.87-0.96)$} & Percent body fat & $0.94(0.89-1.00)$ & $<.001$ \\
\hline & & Total body fat & $0.94(0.89-1.00)$ & $<.001$ \\
\hline & & Intra-abdominal fat area & $0.93(0.88-0.98)$ & 0.051 \\
\hline
\end{tabular}

a The treatment effect ratio was calculated from a general linear model for each biomarker outcome, estimating a parameter whose antilogarithm corresponds to the ratio of adjusted geometric means of the biomarker for the exercise intervention group over the control group: this ratio was assumed to be common at 6 months and 12 months postrandomization. A ratio $<1.0$ indicates lower hormone or SHBG levels in exercisers relative to controls at 6 and 12 months; a ratio $>1.0$ indicates a higher hormone or SHBG levels in exercisers; and a ratio equal to 1.0 indicates no difference between exercisers and controls. The model after-adjustment has one more covariate: change of hypothesized mediator at 6 and 12 months, compared to the model before-adjustment

b $P$ value for the association between mediator and outcome after adjustment for intervention assignment

Sample size $N$ : exercise group, baseline $=160,6$ month $=154,12$ month $=154$; control group, baseline $=160$, 6 month $=154$, 12 month $=156$ 
Table 3 Exercise intervention effects on sex hormones, stratified by potential moderators

\begin{tabular}{|c|c|c|c|c|c|}
\hline \multirow{2}{*}{$\begin{array}{l}\text { Moderator }^{\mathrm{a}} \\
\text { Level }\end{array}$} & \multirow[t]{2}{*}{$n^{\mathrm{b}}$} & \multicolumn{4}{|c|}{ Treatment effect ratio ${ }^{c}(95 \% \mathrm{CI})$} \\
\hline & & Estradiol & Estrone & Testosterone & SHBG \\
\hline \multicolumn{6}{|c|}{ Physical fitness $\left(\mathrm{VO}_{2 \max }\right)$} \\
\hline$\leq 27.5 \mathrm{~mL} / \mathrm{kg} / \mathrm{min}$ & $82 / 80$ & $0.98(0.92-1.04)$ & $0.98(0.92-1.05)$ & $0.97(0.92-1.03)$ & $1.06(1.02-1.09)$ \\
\hline$>27.5$ & $77 / 79$ & $0.89(0.82-0.96)$ & $0.99(0.93-1.05)$ & $1.01(0.95-1.07)$ & $1.03(0.99-1.07)$ \\
\hline$p^{\mathrm{e}}$ & & 0.573 & 0.175 & 0.035 & 0.005 \\
\hline \multicolumn{6}{|l|}{ Age } \\
\hline$\leq 60$ years & $73 / 81$ & $0.91(0.83-0.99)$ & $1.01(0.95-1.07)$ & $1.03(0.97-1.10)$ & $1.04(1.00-1.08)$ \\
\hline$>60$ & $87 / 78$ & $0.96(0.91-1.02)$ & $0.96(0.90-1.03)$ & $0.95(0.90-1.01)$ & $1.04(1.01-1.08)$ \\
\hline$p^{\mathrm{e}}$ & & 0.712 & 0.200 & 0.038 & 0.480 \\
\hline \multicolumn{6}{|c|}{ Time since menopause } \\
\hline$<10$ years & $80 / 69$ & $0.91(0.84-0.99)$ & $1.02(0.96-1.08)$ & $1.02(0.96-1.08)$ & $1.05(1.02-1.09)$ \\
\hline$\geq 10$ & $78 / 90$ & $0.96(0.91-1.02)$ & $0.96(0.90-1.03)$ & $0.95(0.90-1.00)$ & $1.04(1.00-1.08)$ \\
\hline$p^{\mathrm{e}}$ & & 0.673 & 0.776 & 0.609 & 0.477 \\
\hline \multicolumn{6}{|l|}{ Previous HRT use } \\
\hline Yes & $75 / 71$ & $0.92(0.87-0.99)$ & $0.97(0.90-1.04)$ & $0.96(0.90-1.02)$ & $1.02(0.98-1.06)$ \\
\hline No & $84 / 88$ & $0.94(0.87-1.01)$ & $1.00(0.95-1.06)$ & $1.01(0.95-1.07)$ & $1.07(1.03-1.10)$ \\
\hline$p^{\mathrm{e}}$ & & 0.754 & 0.480 & 0.326 & 0.149 \\
\hline \multicolumn{6}{|l|}{ Self-rated health ${ }^{\mathrm{d}}$} \\
\hline Low & $88 / 76$ & $0.93(0.86-1.01)$ & $1.01(0.94-1.07)$ & $0.97(0.91-1.03)$ & $1.05(1.02-1.09)$ \\
\hline High & $71 / 81$ & $0.92(0.86-0.98)$ & $0.97(0.92-1.04)$ & $1.02(0.96-1.08)$ & $1.04(1.00-1.08)$ \\
\hline$p^{\mathrm{e}}$ & & 0.516 & 0.441 & 0.968 & 0.923 \\
\hline \multicolumn{6}{|c|}{ Past year recreational activity } \\
\hline <7 MET-h/week & $87 / 74$ & $0.88(0.82-0.95)$ & $0.96(0.90-1.02)$ & $0.95(0.90-1.01)$ & $1.05(1.01-1.09)$ \\
\hline$\geq 7$ & $73 / 85$ & $0.97(0.90-1.05)$ & $1.01(0.94-1.07)$ & $1.02(0.96-1.09)$ & $1.04(1.00-1.08)$ \\
\hline$p^{\mathrm{e}}$ & & 0.642 & 0.656 & 0.637 & 0.508 \\
\hline \multicolumn{6}{|l|}{ Body mass index } \\
\hline$<28.7 \mathrm{~kg} / \mathrm{m}^{2}$ & $80 / 77$ & $0.95(0.87-1.03)$ & $1.04(0.97-1.11)$ & $1.03(0.97-1.09)$ & $1.03(0.99-1.07)$ \\
\hline$\geq 28.7$ & $80 / 82$ & $0.91(0.86-0.96)$ & $0.94(0.89-1.00)$ & $0.95(0.90-1.01)$ & $1.06(1.02-1.10)$ \\
\hline$p^{\mathrm{e}}$ & & 0.714 & 0.066 & 0.179 & 0.586 \\
\hline \multicolumn{6}{|l|}{ Estradiol } \\
\hline$<10 \mathrm{pg} / \mathrm{mL}$ & $86 / 70$ & $0.97(0.90-1.04)$ & & & \\
\hline$\geq 10$ & $74 / 89$ & $0.90(0.84-0.97)$ & & & \\
\hline$p^{\mathrm{e}}$ & & 0.935 & & & \\
\hline \multicolumn{6}{|l|}{ Estrone } \\
\hline$<32 \mathrm{pg} / \mathrm{mL}$ & $87 / 85$ & & $1.01(0.95-1.08)$ & & \\
\hline$\geq 32$ & $73 / 74$ & & $0.96(0.90-1.02)$ & & \\
\hline$p^{\mathrm{e}}$ & & & 0.180 & & \\
\hline \multicolumn{6}{|l|}{ Testosterone } \\
\hline$<24 \mathrm{ng} / \mathrm{dL}$ & $77 / 82$ & & & $0.99(0.93-1.06)$ & \\
\hline$\geq 24$ & $83 / 77$ & & & $0.99(0.93-1.04)$ & \\
\hline$p^{\mathrm{e}}$ & & & & 0.497 & \\
\hline \multicolumn{6}{|l|}{ SHBG } \\
\hline$<40 \mathrm{nmol} / \mathrm{L}$ & $76 / 85$ & & & & $1.06(1.02-1.10)$ \\
\hline$\geq 40$ & $84 / 74$ & & & & $1.03(0.99-1.06)$ \\
\hline$p^{\mathrm{e}}$ & & & & & 0.764 \\
\hline
\end{tabular}

SHBG sex hormone-binding globulin

${ }^{a}$ Level of potential moderator at baseline

b Number of exercisers/number of controls

c The treatment effect ratio was calculated from a general linear model for each biomarker outcome, estimating a parameter whose anti-logarithm corresponds to the ratio of adjusted geometric means of the biomarker for the exercise intervention group over the control group: this ratio was assumed to be common at 6 months and 12 months postrandomization. A ratio $<1.0$ indicates lower hormone or SHBG levels in exercisers relative to controls at 6 and 12 months; a ratio $>1.0$ indicates higher hormone or SHBG levels in exercisers; and a ratio equal to 1.0 indicates no difference between exercisers and controls

d Self-rated health measured by self-administered questionnaire using the SF-36 scale (range, 0-100) where low was a score of $<82$ and high was $\geq 82$

e Statistical significance of the interaction term between intervention group and the potential moderator. Note that all moderators except for previous HRT use were treated as continuous variables when calculating this $p$ value for heterogeneity 
fitness levels than among women with higher fitness (continuous, $p=0.005$ ). For testosterone, intervention effects were comparable across dichotomized subgroups; however, fitness (continuous, $p=0.035$ ) and age (continuous, $p=0.038$ ) appeared to moderate the intervention effect in general linear models.

In exploratory analyses, no baseline factor examined as a moderator was significantly correlated with adherence (measured as average weekly duration of exercise) among exercisers.

\section{Discussion}

This intervention study investigated potential factors that may mediate and moderate the relation between endogenous sex hormone changes and physical activity among postmenopausal women. This important research question has not been formally examined in previous randomized trials. We found that exercise effects on SHBG and estradiol change were attenuated with adjustment for total and percent body fat change and somewhat attenuated with adjustment for intra-abdominal fat change. Intervention effects on SHBG levels were unaffected by adjustment for insulin change. Baseline physical fitness that appeared to moderate the intervention effects on testosterone and SHBG levels and age seemed to moderate the intervention effects on testosterone levels.

Overall fat loss appeared to mediate SHBG change since, in general linear models, SHBG change was associated with changes in all measures of adiposity, and decreases in percent body fat, total body fat, and intraabdominal fat each explained most of the total effect of exercise on SHBG change. In another RCT of postmenopausal women, McTiernan et al. [19] similarly found that the greatest 12-month increases in SHBG levels occurred in the subgroup of women who lost $>2 \%$ body fat, regardless of group assignment. A recent RCT by Monninkhof et al. [20] did not find a significant effect of exercise on SHBG change over 12 months, possibly because the average decrease in fat mass among exercisers $(0.8 \mathrm{~kg})$ was lower than in our study $(2.4 \mathrm{~kg}$; ref [8]).

We explored insulin reduction as a possible mediator of exercise-induced increases in SHBG levels since insulin lowers SHBG synthesis in the liver [5]. We found that although exercisers experienced a greater decrease in serum insulin concentrations than controls [7], this change did not explain the intervention effect for SHBG. Yet, in exploratory analyses, insulin change correlated with SHBG change in the control group (but not exercisers) and was significantly associated with SHBG change after controlling for intervention effects. Overall, these analyses imply that while insulin changes may have altered SHBG concentrations in some women in the trial, this change does not explain why SHBG changes were greater for exercisers than for controls.

Relatively consistent RCT evidence now points to fat loss as a mediator of exercise-induced estradiol changes. From stratified analyses, McTiernan et al. [19] concluded that combined exercise and fat loss of at least $0.5 \%$ may be required to decrease total and free estradiol concentrations; exercise without this level of fat loss did not affect estradiol concentrations. Monninkhof et al. [20] found complete attenuation of an exercise effect on free estradiol levels after adjusting for body fat change, similarly implicating a fat loss mechanism. Our adjusted models showed that overall body fat loss and exercise contributed independently to free and total estradiol changes over 12 months; only a portion of the total effect of the intervention on estradiol change was explained by fat loss. We also found that intra-abdominal fat area did not appear to mediate total estradiol changes, which is of interest to note given that intra-abdominal fat in postmenopausal women relates adversely to adipokine and inflammation levels [21], which are causally related to several chronic diseases [22, 23]. In a comprehensive 2007 report on cancer prevention [2], overall body fatness and abdominal fatness were concluded to be "convincingly" and "probably" associated with an increased risk of postmenopausal breast cancer, respectively.

From a biologic perspective, exercise could lower estradiol concentrations in a variety of ways postmenopause, with loss of body fat proposed as a key mechanism. After menopause, adipose tissue serves as a reservoir of estrogen biosynthesis [24, 25]. Furthermore, several blood biomarkers that occur at higher levels in overweight and obese states such as leptin [26] and IL-6 and TNF- $\alpha$ [27] may induce or stimulate this biosynthesis in some women [28]. It is also possible that adiposity change was merely a surrogate marker of the true mediator of estradiol (and SHBG) changes. Although error in measurement of adiposity change could explain why we did not find that it wholly mediated exercise-induced estradiol changes, the use of valid and reliable measures makes this possibility less plausible. Mechanisms whereby exercise could affect estradiol levels independently of fat loss are as of yet unknown.

To our knowledge, no previous RCT in older women has formally tested moderation of exercise-induced SHBG or sex hormone changes. However, RCTs of 12-month aerobic exercise interventions described by McTiernan et al. [29] and Irwin et al. [30] explored potential moderators of adiposity change, which is relevant to this discussion. Neither McTiernan et al. [29], studying 100 women with a mean age of 54 years, nor Irwin et al. [30], studying 173 
postmenopausal women, found statistically significant effect modification by baseline BMI or age. Although we found evidence of moderating effects of age and baseline fitness on testosterone changes, the magnitude of this moderation did not appear to be great since treatment effect ratios were close to 1.0 in subgroups of these characteristics and not statistically significant. The strongest evidence of moderation was for baseline fitness level and SHBG change, in which intervention effects on SHBG appeared to be stronger in women who were less fit at baseline. Exploratory analyses suggested that effect modification did not seem to be explained by differences in adherence but could be explained by differences in fitness change or another factor for which fitness change may be a surrogate. Women with lower baseline fitness level experienced greater improvements in fitness over the 12-month intervention.

There are several limitations to our analyses. First, we did not address all factors that could theoretically impact individual responsiveness to exercise (e.g., genotype) in our moderators analysis. Further limitations apply specifically to the analysis of mediators [31]. For example, mediation might differ across subgroups of exercising women, but we did not have the power to explore this possibility within our data. Our findings may not be generalizable to all postmenopausal women as our study population was free of any major co-morbidities, predominantly Caucasian and educated, and included normal weight women as well as overweight and obese women $[6,8]$.

In conclusion, these analyses suggest possible mediation of exercise-induced changes in estradiol and SHBG concentrations by loss of overall body fat. Our data further imply that other biologic mechanisms related to exercise were also driving estradiol changes, potentially lowering postmenopausal breast cancer risk, and thus, there is a need to identify those mechanisms. While strong biologic plausibility supports mediation of estradiol changes through fat loss, the understanding of how fat loss relates to SHBG concentrations is less clear. Whether fat loss mediates those changes, or represents a surrogate marker of the true mediator, warrants further study. We found no evidence supporting insulin change as a mediator of exercise-induced changes in SHBG levels. We identified baseline physical fitness and age as possible moderators of exercise-induced testosterone and SHBG changes. This research provides compelling evidence among postmenopausal women of how the relation between physical activity and endogenous sex hormone changes is mediated by body fat loss and the first evidence of moderation by physical fitness and age. Future research will be needed in other populations to corroborate and expand these latter findings.
Acknowledgments The study setup was done by Kim van der Hoek and Marla Orenstein, and the Study Coordinators were Rosemary Crosby and Ame-Lia Tamburrini. The Fitness Centre Managers were Ben Wilson, Lisa Workman, and Diane Cook. The Exercise Trainers were Shannon Hutchins, Kathy Traptow, Shannon Brown, Susan Daniel, Parissa Gillani, Stephanie Sanden, Karen Mackay, and Sandra Olsen. Data preparation was done by Sandra Blitz and Sony Brar. This study was funded by a research grant (\#017468) from the Canadian Breast Cancer Research Alliance. Dr. Friedenreich was funded by career awards from Canadian Institutes of Health Research and the Alberta Heritage Foundation for Medical Research. Dr. Courneya is supported by the Canada Research Chairs Program.

Open Access This article is distributed under the terms of the Creative Commons Attribution Noncommercial License which permits any noncommercial use, distribution, and reproduction in any medium, provided the original author(s) and source are credited.

\section{References}

1. Lynch BM, Neilson HK, Friedenreich CM (2011) Physical activity and breast cancer prevention. Recent Results Cancer Res 186:13-42

2. World Cancer Research Fund the American Institute for Cancer Research (2007) Food, nutrition, physical activity, and the prevention of cancer: a global perspective. American Institute for Cancer Research, Washington, DC

3. Key T, Appleby P, Barnes I, Reeves G (2002) Endogenous sex hormones and breast cancer in postmenopausal women: reanalysis of nine prospective studies. J Natl Cancer Inst 94(8):606-616

4. Neilson HK, Friedenreich CM, Brockton NT, Millikan RC (2009) Physical activity and postmenopausal breast cancer: proposed biologic mechanisms and areas for future research. Cancer Epidemiol Biomarkers Prev 18(1):11-27

5. Plymate SR, Jones RE, Matej LA, Friedl KE (1988) Regulation of sex hormone binding globulin (SHBG) production in Hep G2 cells by insulin. Steroids 52(4):339-340

6. Friedenreich CM, Woolcott CG, McTiernan A et al (2010) Alberta physical activity and breast cancer prevention trial: sex hormone changes in a year-long exercise intervention among postmenopausal women. J Clin Oncol 28(9):1458-1466

7. Friedenreich CM, Neilson HK, Woolcott CG et al (2011) Changes in insulin resistance indicators, insulin-like growth factors, and adipokines in a year-long trial of aerobic exercise in postmenopausal women. Endocr Relat Cancer 18(3):357-369

8. Friedenreich CM, Woolcott CG, McTiernan A et al (2010) Adiposity changes after a 1-year aerobic exercise intervention among postmenopausal women: a randomized controlled trial. Int J Obes (Lond) 35:427-435

9. Kraemer HC, Wilson GT, Fairburn CG, Agras WS (2002) Mediators and moderators of treatment effects in randomized clinical trials. Arch Gen Psychiatry 59(10):877-883

10. Mackinnon DP, Fairchild AJ (2009) Current directions in mediation analysis. Curr Dir Psychol Sci 18(1):16

11. British Columbia Ministry of Health, Canadian Society for Exercise Physiology (2002) Physical activity readiness medical examination

12. Friedenreich CM, Courneya KS, Neilson HK et al (2006) Reliability and validity of the Past Year Total Physical Activity Questionnaire. Am J Epidemiol 163(10):959-970

13. American College of Sports Medicine (2000) ACSM's guidelines for exercise testing and prescription, 6th edn. Lippincott Williams \& Wilkins, Philadelphia, PA 
14. Goebelsmann U, Horton R, Mestman JH et al (1973) Male pseudohermaphroditism due to testicular 17 -hydroxysteroid dehydrogenase deficiency. J Clin Endocrinol Metab 36(5):867879

15. Goebelsmann U, Bernstein GS, Gale JA et al (1979) Serum gonadotropin, testosterone, estradiol and estrone levels prior to and following bilateral vasectomy. In: Lepow IH, Crozier R (eds) Vasectomy: immunologic and pathophysiologic effects in animals and man. Academic Press, New York, p 165

16. Probst-Hensch NM, Ingles SA, Diep AT et al (1999) Aromatase and breast cancer susceptibility. Endocr Relat Cancer 6(2): $165-173$

17. Rinaldi S, Geay A, Dechaud H et al (2002) Validity of free testosterone and free estradiol determinations in serum samples from postmenopausal women by theoretical calculations. Cancer Epidemiol Biomarkers Prev 11(10 Pt 1):1065-1071

18. Ware JE, Sherbourne CD (1992) The MOS 36-item Short-Form Health Survey (SF-36). I. Conceptual framework and item selection. Med Care 30:473-483

19. McTiernan A, Tworoger SS, Ulrich CM et al (2004) Effect of exercise on serum estrogens in postmenopausal women: a 12-month randomized clinical trial. Cancer Res 64(8):2923-2928

20. Monninkhof EM, Velthuis MJ, Peeters PH, Twisk JW, Schuit AJ (2009) Effect of exercise on postmenopausal sex hormone levels and role of body fat: a randomized controlled trial. J Clin Oncol 27(27):4492-4499

21. Lee CG, Carr MC, Murdoch SJ et al (2009) Adipokines, inflammation, and visceral adiposity across the menopausal transition: a prospective study. J Clin Endocrinol Metab 94(4): 1104-1110
22. Pradhan AD, Manson JE, Rossouw JE et al (2002) Inflammatory biomarkers, hormone replacement therapy, and incident coronary heart disease: prospective analysis from the Women's Health Initiative observational study. JAMA 288(8):980-987

23. Pradhan AD, Manson JE, Rifai N, Buring JE, Ridker PM (2001) C-reactive protein, interleukin 6 , and risk of developing type 2 diabetes mellitus. JAMA 286(3):327-334

24. Simpson ER, Merrill JC, Hollub AJ, Graham-Lorence S, Mendelson CR (1989) Regulation of estrogen biosynthesis by human adipose cells. Endocr Rev 10(2):136-148

25. Grodin JM, Siiteri PK, MacDonald PC (1973) Source of estrogen production in postmenopausal women. J Clin Endocrinol Metab 36(2):207-214

26. Magoffin DA, Weitsman SR, Aagarwal SK, Jakimiuk AJ (1999) Leptin regulation of aromatase activity in adipose stromal cells from regularly cycling women. Ginekol Pol 70(1):1-7

27. Purohit A, Reed MJ (2002) Regulation of estrogen synthesis in postmenopausal women. Steroids 67(12):979-983

28. Maccio A, Madeddu C, Mantovani G (2009) Adipose tissue as target organ in the treatment of hormone-dependent breast cancer: new therapeutic perspectives. Obes Rev 10(6):660-670

29. McTiernan A, Sorensen B, Irwin ML et al (2007) Exercise effect on weight and body fat in men and women. Obesity (Silver Spring) 15(6): 1496-1512

30. Irwin ML, Yasui Y, Ulrich CM et al (2003) Effect of exercise on total and intra-abdominal body fat in postmenopausal women: a randomized controlled trial. JAMA 289(3):323-330

31. Bullock JG, Green DP, Ha SE (2010) Yes, but what's the mechanism? (don't expect an easy answer). J Pers Soc Psychol 98(4):550-558 